Arch. Tierz., Dummerstorf 47 (2004) 6, 565-574

Aus dem Institut für Tierzucht und Haustiergenetik, Fachgebiet Tierhaltung und Haltungsbiologie, der Justus-Liebig-Universität Gießen

STEFAN ROHRMANN und STEFFEN HOY

\title{
Ethologische Untersuchungen zur Beschäftigung von Ebern in Besamungsstationen mit Stroh ${ }^{1)}$
}

\author{
Herrn Prof. Dr. habil. Peter Glodek zum 70. Geburtstag gewidmet
}

\begin{abstract}
Title of the paper: Ethological investigations on occupation with the bedding of boars in Artificial Insemination stations

Continuous video recordings with infrared video technique and computer supported analysis with OBSERVER program took place with 78 Piétrain boars in three A.I. stations during $3 \times 24$ hours. The mean percentage of occupation with the bedding during 24 hours was $7.4 \pm 4.4 \%$ (= 106.6 minutes) with a minimum of $1.5 \%$ and a maximum of $18.6 \%$. The factors A.I. station, season and pen size had a significant influence on occupation with straw as bedding material. The percentage of occupation with bedding in winter was $1.8 \%$ higher than in summer. The percentage of occupation with bedding increased with increasing pen size (from $5.5 \%$ to $11.9 \%$ ). The differences between the A.I. stations (between $6.4 \%$ and $10.1 \%$ on average) were caused by management, especially by the frequency of the timers feeding, bedding and watering. The more frequent the boars were caused to stand up by feeding, bedding and watering the higher was the percentage of occupation with the bedding.
\end{abstract}

Key Words: boar, behaviour, occupation, straw, A.I. station

\section{Zusammenfassung}

Kontinuierliche Verhaltensaufzeichnungen mittels Infrarot-Videotechnik und die computergestützte Auswertung am OBSERVER/Video Tape Analysis System bei 78 Piétrain-Ebern in drei Besamungsstationen über je 3 x $24 \mathrm{~h}$ ergaben, dass der durchschnittliche prozentuale Anteil der Beschäftigung mit Stroh in 24 Stunden 7,4 \pm 4,4 \% (= 106,6 Minuten) mit einem Minimum von 1,5 \% und einem Maximum von 18,6 \% betrug. Die Faktoren Besamungsstation, Jahreszeit und Buchtengröße hatten einen signifikanten Einfluss auf die Beschäftigung mit Stroh. Im Winter war der Anteil der Beschäftigung um 1,8 \% höher als im Sommer. Mit zunehmender Buchtengröße stieg die Zeitdauer der Beschäftigung (von 5,5 \% auf 11,9 \%). Die Unterschiede zwischen den Besamungsstationen (zwischen 6,4 \% und 10,1\% im Mittel) waren durch das Management, insbesondere durch die Häufigkeit der Zeitgeber Füttern, Einstreuversorgung und Tränken, bedingt. Je häufiger die Tiere durch Füttern, Einstreuen oder Tränken veranlasst wurden aufzustehen, um so höher war der prozentuale Anteil der Beschäftigung.

Schlüsselwörter: Eber, Verhalten, Beschäftigung, Stroh, Besamungsstation

\section{Einleitung}

Die EU-Richtlinie 2001/93/EG fordert den ständigen Zugang zu ausreichenden Mengen an Materialien, die die Schweine untersuchen und bewegen können, wie z.B.

1) Die Autoren danken dem Förderverein Biotechnologieforschung der Deutschen Schweineproduktion e.V. für die freundliche Unterstützung. 
Stroh, Heu, Holz, Sägemehl, Pilzkompost, Torf oder eine Mischung dieser Materialien, durch die die Gesundheit der Tiere nicht gefährdet werden kann.

Das Anbieten von Stroh als Beschäftigungsmaterial wirkt der Reizarmut eines Haltungssystems entgegen und kann zur Verbesserung der Tiergerechtheit beitragen (BUCHENAUER, 1986; GRAUVOGL, 1987; MARX und BUCHHOLZ, 1991). Da das praxisübliche Futter normalerweise in relativ kurzer Zeit gefressen wird, sind die Schweine dann zwar satt, haben aber ihre Verhaltensbedürfnisse in Bezug auf Futtersuche und Futterbearbeitung noch nicht befriedigt (WECHSLER, 1997). Auch dem Erkundungsverhalten der Schweine kann durch das Vorhandensein von Stroh Rechnung getragen werden; der Einsatz von Stroh kann so dazu beitragen, durch Beschäftigungsarmut ausgelöste Verhaltensabweichungen $\mathrm{zu}$ verhindern (VON BORELL et al., 2002).

Die Auswertung der Literatur ergab, dass mit einer Ausnahme keine quantitativen Aussagen zum Verhalten von Ebern vorliegen. Lediglich in einer Untersuchung aus den Niederlanden fanden BRUININX et al. (1998), dass die Eber im Mittel zwischen $80 \%$ und $90 \%$ des Tages liegend verbrachten und dass die Liegezeiten im Sommer länger waren als in der Winterperiode. Bei der Analyse der Liegedauer in Bezug auf unterschiedliche Haltungssysteme (Kastenstände, Buchten mit Teilspaltenboden und stroheingestreute Buchten) ergab sich kein einheitliches Bild. Das Ziel der eigenen Untersuchungen bestand darin, die häufig emotional geführte Diskussion um Stroh als Einstreu- und Beschäftigungsmaterial zu versachlichen und quantitative Ergebnisse zur Beschäftigung unter Berücksichtigung verschiedener Umweltfaktoren vorzulegen.

Material und Methoden

Die Verhaltensuntersuchungen fanden in drei Besamungsstationen mit insgesamt über 600 Eberplätzen statt, die auf jeweils mehrere Stallabteile pro Station verteilt waren. Die Tiere wurden je nach Bedarf durchschnittlich ein- bis zweimal pro Woche zur Absamung herangezogen. Alle in den Stationen A, B und C gehaltenen Eber befanden sich in Einzelhaltung mit plan befestigtem Fußboden und Stroheinstreu. Jeder Eber hatte über vergitterte Buchtenwände Sicht- und Schnauzenkontakt mit anderen Ebern. Die Flächengröße der Buchten variierte zwischen 5 und $8 \mathrm{~m}^{2}$. Einige wenige Buchten in Station C hatten eine Größe von mehr als $13 \mathrm{~m}^{2}$. Die Eber der Stationen A und C wurden zweimal täglich gefüttert, in Station B gab es nur eine Fütterungszeit am Tag. Trinkwasser stand zur freien Verfügung; lediglich in Station A wurde während der Sommer-Untersuchung dreimal täglich der Trog mit Wasser gefüllt. Die Entmistung der Eberbuchten fand je nach Station und Stallabteil zwei- bis dreimal pro Woche statt. Die Verhaltensuntersuchungen erfolgten mittels Infrarot-Videotechnik und Langzeitrecording stets kontinuierlich über 24 Stunden. Anschließend wurde mit Hilfe eines Time-Code-Generators ein Zeitcode im Sekundentakt auf die Videobänder aufgespielt; dies war Voraussetzung für die computergestützte Auswertung der Verhaltensaufnahmen, die lückenlos und sekundengenau unter Einsatz des Observer/ Video Tape Analysis Systems stattfand.

Für die Infrarot-Videoaufnahmen und die anschließende Auswertung kam folgende Technik zum Einsatz (HOY, 1998a, b):

- Restlichtkamera WV-BP 500 (Panasonic)

- Langzeit-Videorecorder AG 6024 HE (Panasonic)

- Infrarotstrahler mit Netzteil WFL-I-LED $30 \mathrm{~W}$ 
- Monitor WV-BM 80

- Videokassetten 180 min

- Time-Code-Generator AEC-Box 28

- Observer/Video Type Analysis System (Fa. Noldus, NL)

Insgesamt gingen die Verhaltensbeobachtungen von 78 Ebern in die Untersuchung ein. Es wurden ausschließlich Eber der Rasse Piétrain ausgewählt, da diese Rasse mit Abstand den größten Anteil aller in den Stationen gehaltenen Eber ausmachte. Die Hälfte der beobachteten Tiere waren Jungeber ( $n=39$; $\leq 24$ Monate), die andere Hälfte ( $\mathrm{n}=39$ ) war älter als 2 Jahre (Alteber, ROHRMANN und HOY, 2004a).

In jeder der drei Besamungsstationen fand jeweils eine Untersuchungsperiode im Sommerhalbjahr und eine im Winterhalbjahr statt. Das Verhalten der für die Untersuchungen ausgewählten Tiere wurde kontinuierlich an je drei aufeinanderfolgenden Tagen (nahezu ausschließlich Montag bis Donnerstag) aufgezeichnet. Für jeden Eber gingen demnach $3 \times 24 \mathrm{~h}=72$ Stunden Beobachtungszeit in die Auswertung ein. Dabei wurden folgende ausgewählte Verhaltensparameter der Eber quantitativ auf Sekundenbasis erfasst:

- aktives und passives Liegen (aktives Liegen: Liegen in Bauchlage, teilweise im Stroh wühlend; passives Liegen: Liegen in Seitenlage ohne andere Verhaltensweisen)

- Laufen und Stehen

- Beschäftigung mit Stroh

- Beschäftigung mit Artgenossen in der Nachbarbucht (ROHRMANN und HOY, 2004b).

Die vorliegende Auswertung konzentriert sich auf die Beschäftigung der Eber mit Stroh. Die Daten eines jeden Ebers für die drei aufeinanderfolgenden Tage wurden zusammengefasst und als prozentuale Anteile (der Beschäftigung mit Stroh) bezogen auf die Gesamtbeobachtungszeit von 72 Stunden (= 259.200 Sekunden) zur weiteren statistischen Bearbeitung in eine Excel-Datei eingetragen.

Da die Normalverteilung der Verhaltensdaten gegeben war (Kolomogorov-SmirnovTest), konnte anschließend die Schätzung der LSQ-Mittelwerte für den prozentualen Zeitanteil „Beschäftigung mit Stroh (in 24 Stunden)“ mittels univariater Varianzanalyse erfolgen. Dazu wurde folgendes Modell verwendet:

Beobachtungswert $_{\mathrm{ijkl}}=\mu+$ Station $_{\mathrm{i}}+$ Buchtenkategorie $_{\mathrm{j}}+$ Jahreszeit $_{\mathrm{k}}$

$$
+\left(\text { Station x Jahreszeit) }{ }_{i k}+b\left(\text { Alter }_{1}-\overline{\text { Alter }}\right)+e_{i j k l}\right.
$$

Neben der Kovariable Alter gingen die übrigen untersuchten Einflussfaktoren Besamungsstation, Buchtengröße und Jahreszeit sowie die Interaktion Station x Jahreszeit als fixe Effekte in das statistische Modell ein. Die Interaktion Buchtengröße x Jahreszeit wurde zunächst getestet, da sich aber keine Signifikanzen ergaben, nicht im Modell berücksichtigt. Die statistische Bearbeitung erfolgte mit SPSS 11.5.

\section{3. $\quad$ Ergebnis}

Die deskriptive Statistik für die Originalwerte der 78 Eber ergab für den prozentualen Anteil der Beschäftigung mit Stroh in 24 Stunden einen Wert von 7,4 \% \pm 4,4 \% ( $\overline{\mathrm{x}} \pm$ s), d.h. im Mittel beschäftigten sich die Tiere pro Tag 106,6 Minuten mit dem Stroh. Zwischen den Ebern traten gravierende Unterschiede hinsichtlich des Beschäfti- 
gungsanteils in 24 Stunden zutage - mit einem Minimum von 1,5 \% (= 21,6 min in 24 h) und einem Maximum von 18,6 \% (= 267,8 min in 24 h).

Mit Hilfe der univariaten Varianzanalyse wurde nachgewiesen, dass die Faktoren Besamungsstation, Jahreszeit und Buchtengröße einen signifikanten Einfluss auf die Zielgröße „Beschäftigung mit Stroh“ haben (Tabelle). In der Besamungsstation A war der Prozentsatz an Beschäftigung mit Stroh in 24 Stunden um 2,4 \% bzw. 3,7 \% signifikant höher als in den Stationen B und C. Im Winter war im Mittel aller Tiere die Beschäftigung um 1,8 \% länger als im Sommer (bezogen auf 24 Stunden). Mit zunehmender Buchtengröße stieg die prozentuale Dauer der Beschäftigung von 5,5 \% auf 11,9 \%. Alle Angaben beziehen sich auf ein im Modell korrigiertes Alter der Eber von 28,1 Monaten. Das Alter selbst hatte keinen statistisch gesicherten Einfluss auf die Beschäftigungsdauer. Mit zunehmendem Alter der Tiere verringerte sich allerdings tendenziell der Beschäftigungsanteil in 24 Stunden von 8,0 \% im Alter von durchschnittlich 16 Monaten auf 6,8 \% bei im Mittel 40 Monate alten Ebern.

Tabelle

Ergebnisse der univariaten Varianzanalyse: Einfluss von Besamungsstation, Jahreszeit und Buchtengröße auf den prozentualen Anteil der Beschäftigung mit Stroh von Besamungsebern in 24 Stunden (bei einem Alter der Eber im Modell von 28,1 Monaten) Results of univariate analysis of variance: Influence of A.I. station, season and pen size on percentage of occupation with straw in boars during 24 hours (at an age of boars in the model of 28.1 months)

\begin{tabular}{lccc}
\hline Faktor & & $\begin{array}{c}\text { Prozentsatz der Beschäftigung mit Stroh } \\
\text { in 24 Stunden (\%) }\end{array}$ \\
\hline Besamungsstation & A & 10,1 & $\mathrm{p}<0,05$ \\
\hline Jahreszeit & B & 7,7 & $\mathrm{p}<0,05$ \\
& $\mathrm{C}$ & 7,2 & $\mathrm{p}<0,05$ \\
\hline Buchtengröße & Sommer & 9,0 & $\mathrm{p}<05$ \\
& Winter & 7,2 & 7,7 \\
\end{tabular}

Da in der univariaten Varianzanalyse ein hochsignifikanter Einfluss der Wechselwirkung aus Station x Jahreszeit nachgewiesen werden konnte, wurden die Tagesgänge der Verhaltensweise „Beschäftigung mit Stroh“ getrennt für die drei Besamungsstationen und jeweils für die Sommer- und Winterperiode berechnet. Die Ergebnisse lassen eine deutliche - wenngleich von Station zu Station verschiedene - Tagesrhythmik des Verhaltens erkennen (Abb. 1 bis 6), die durch die Zeitgeber Füttern, Einstreuen und (nur in Station A und nur im Sommer) Tränken beeinflusst wird. Insbesondere in Station A im Sommer mit der höchsten Anzahl an Zeitgebern (3 x Tränken, 2 x Füttern, 1 x Einstreuen im Tagesverlauf) trat der höchste prozentuale Anteil an Beschäftigung mit Stroh in 24 Stunden auf (Abb. 1). Die beiden höchsten Stundenwerte mit jeweils über 30 \% lagen in unmittelbarer Nähe zur morgendlichen bzw. mittäglichen Tränkzeit. Nach dem Einstreuen stieg der Prozentsatz an Beschäftigung pro Stunde, wobei zu beachten ist, dass die Aufzeichnung über 72 Stunden (= 3 Tage) stattfand, das Einstreuen im Unterschied zu den täglichen Fütterungs- und Tränkzeiten jedoch nur einmal während dieser Zeit erfolgte. Auch in unmittelbarer zeitlicher Nähe zu den Fütterungszeiten waren deutliche Peaks der Beschäftigung mit Stroh zu erkennen. 


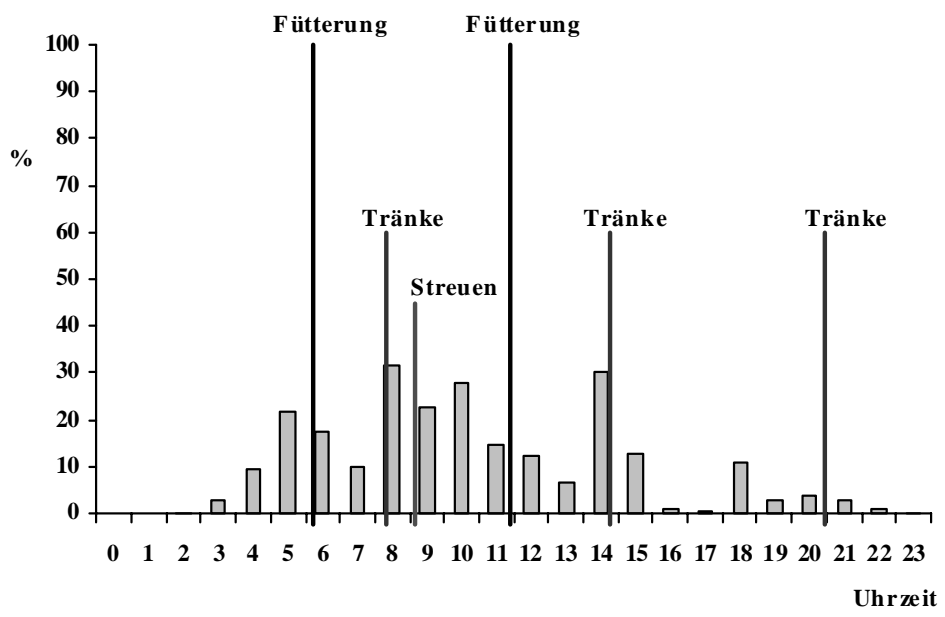

Abb. 1: Prozentualer Anteil der Beschäftigung mit Stroh im Tagesgang (Station A, Sommer, 12 Eber, 72 Stunden Beobachtung) (Percentages of occupation with the bedding during 24 hours - A.I. station A, summer, 12 boars, 72 hours of observation)

Im Winter entfiel in der Station A die dreimal tägliche Tränke, und die Eber hatten wie auch in den anderen beiden Besamungsstationen - ständig Zugang zu Tränkwasser. Der Prozentsatz an Beschäftigung mit Stroh - insgesamt und pro Stunde - war deutlich niedriger als im Sommer. Die Zeitgeberfunktion der Fütterung und der Einstreuversorgung ließ sich erkennen, wenngleich es nicht zu solch markanten Peaks wie in der Sommersituation kam (Abb. 2).

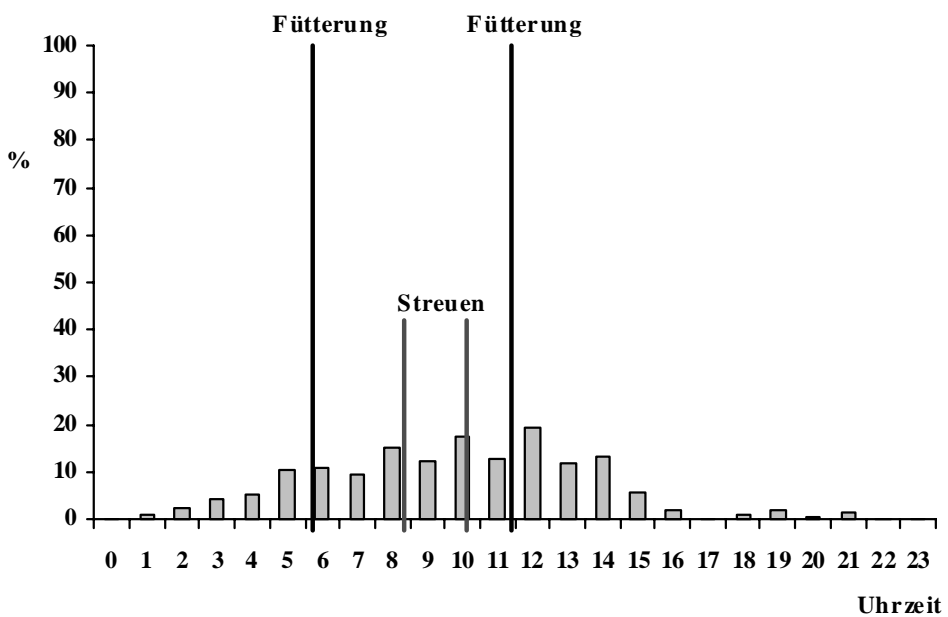

Abb. 2: Prozentualer Anteil der Beschäftigung mit Stroh im Tagesgang (Station A, Winter, 12 Eber, 72 Stunden Beobachtung) (Percentages of occupation with the bedding during 24 hours - A.I. station A, winter, 12 boars, 72 hours of observation)

Über 10 Stunden hinweg (nach 16 Uhr bis gegen 3 Uhr morgens) war die Beschäftigung mit Stroh außerordentlich niedrig. In Station B beschäftigten sich die Eber im Sommer nur in relativ geringem Umfang mit Stroh (Abb. 3). Die höchsten Stundenwerte traten in zeitlicher Nähe zu den Zeitgebern Fütterung und Einstreuversorgung auf. Im Winter war der Zeitanteil der Beschäftigung mit Stroh in 
dieser Station höher als im Sommer. Gegen 11 Uhr und damit wiederum zeitlich nahe zum Füttern und Einstreuen trat der maximale Stundenwert von nahezu 25 \% (etwas weniger als 15 Minuten) auf (Abb. 4).

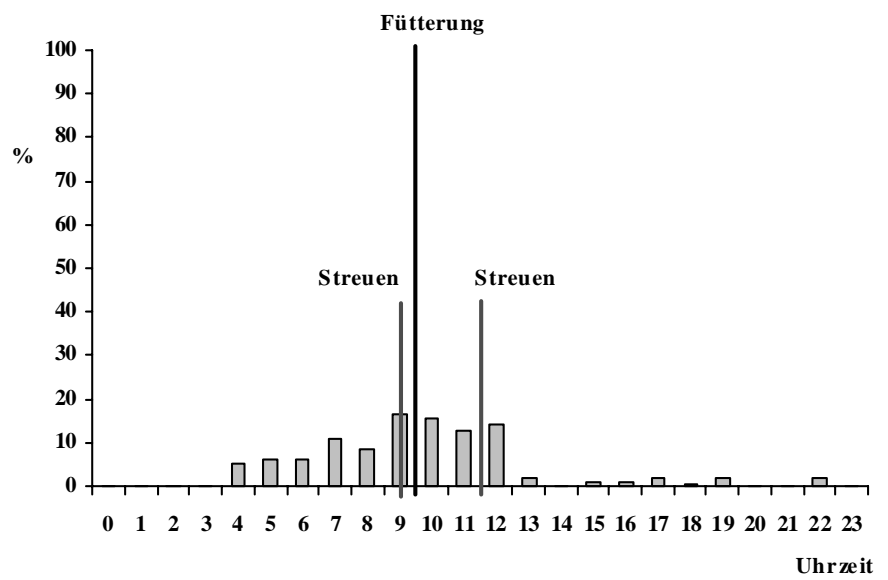

Abb. 3: Prozentualer Anteil der Beschäftigung mit Stroh im Tagesgang (Station B, Sommer, 12 Eber, 72 Stunden Beobachtung) (Percentages of occupation with the bedding during 24 hours - A.I. station B, summer, 12 boars, 72 hours of observation)

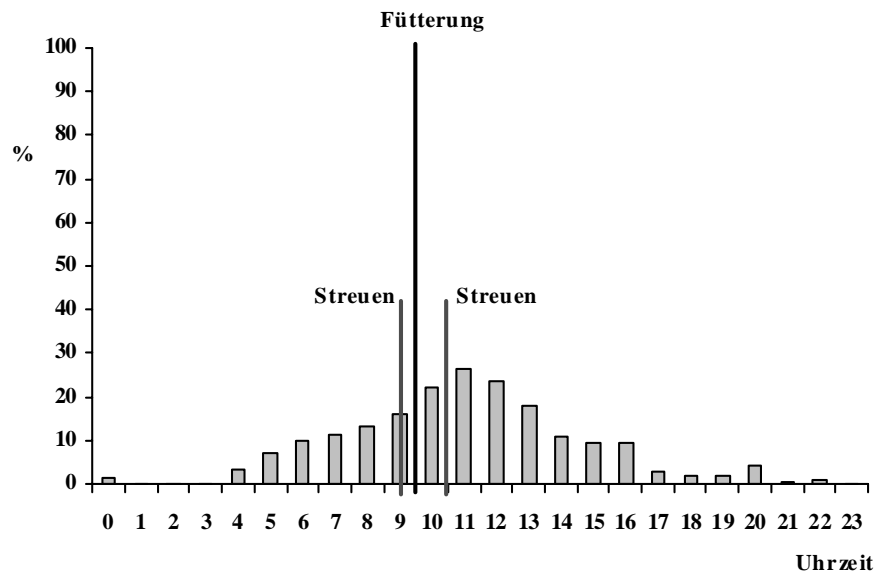

Abb. 4: Prozentualer Anteil der Beschäftigung mit Stroh im Tagesgang (Station B, Winter, 12 Eber, 72 Stunden Beobachtung) (Percentages of occupation with the bedding during 24 hours - A.I. station B, winter, 12 boars, 72 hours of observation)

Ähnlich wie in Besamungsstation 2 war auch in Station 3 die prozentuale Beschäftigung mit Stroh im Sommer niedrig und geringer als im Winter (Abb. 5 und 6). Aus technischen Gründen konnten hierbei nur die Daten von je 7 Ebern berücksichtigt werden. Über 14 Stunden hinweg (von 15 Uhr bis 4 Uhr) war die stündliche Quote an Beschäftigung kaum wahrnehmbar. Zwischen 5 Uhr und 14 Uhr wurden stündliche Beschäftigungsanteile von 7 bis 13 \% nachgewiesen. Das entspricht etwa 4,2 bis 7,8 Minuten pro Stunde. 


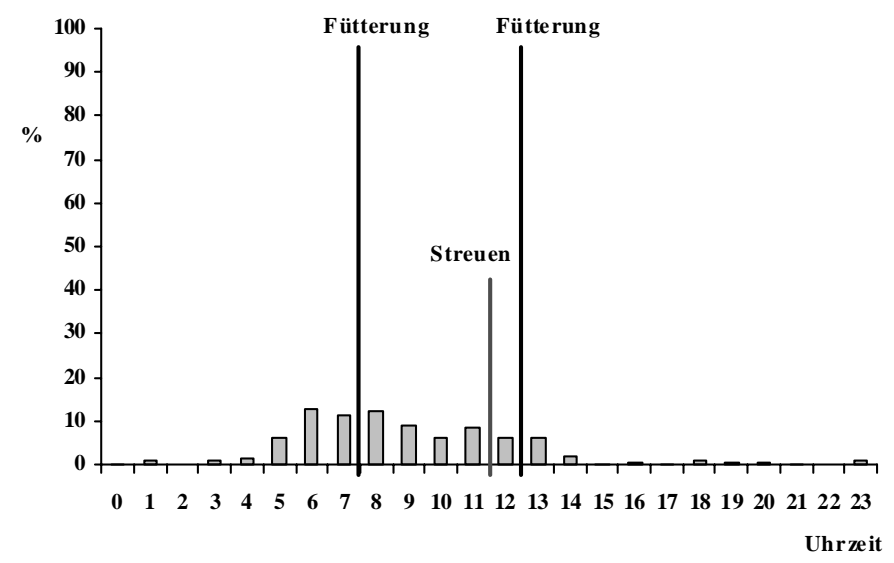

Abb. 5: Prozentualer Anteil der Beschäftigung mit Stroh im Tagesgang (Station C, Sommer, 7 Eber, 72 Stunden Beobachtung) (Percentages of occupation with the bedding during 24 hours - A.I. station C, summer, 7 boars, 72 hours of observation)

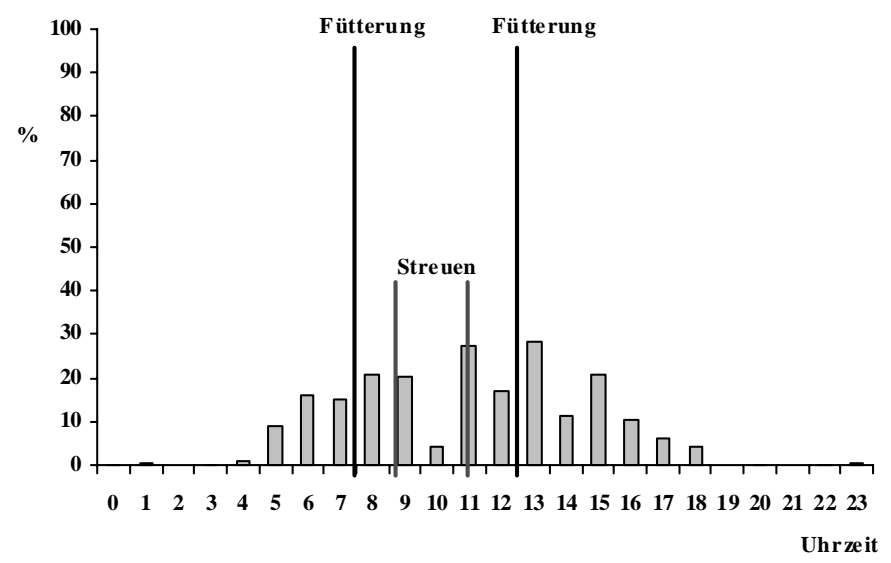

Abb. 6: Prozentualer Anteil der Beschäftigung mit Stroh im Tagesgang (Station C, Winter, 7 Eber, 72 Stunden Beobachtung) (Percentages of occupation with the bedding during 24 hours - A.I. station C, winter, 7 boars, 72 hours of observation)

Im Winter trat die Zeitgeberfunktion von Fütterung und Einstreuversorgung stärker als im Sommer hervor (Abb. 6). Es waren zwei Phasen gesteigerter Beschäftigung mit Stroh - zwischen 5 und 9 Uhr und zwischen 11 und 15 Uhr - zu unterscheiden. Ab 19 Uhr bis 4 Uhr tendierte die Strohbeschäftigung gegen Null.

4. Diskussion

Wenngleich die Bedeutung von Stroh als Beschäftigungsmaterial für Schweine (Ferkel, Mastschweine, Sauen) von verschiedenen Autoren hervorgehoben wird (u.a. HORSTMEYER und VALLBRACHT, 1990; JACKISCH et al., 1996; KRÖTZL et al., 1993; KRESS et al., 1999; STUBBE, 2000), fällt auf, dass vergleichsweise wenige quantitative Untersuchungen dazu vorliegen. Bei Besamungsebern existieren mit einer Ausnahme (BRUININX et al., 1998) überhaupt keine Ergebnisse quantitativer ethologischer Untersuchungen im zugänglichen Schrifttum. Viele Verhaltensuntersuchungen 
wurden als Direktbeobachtungen - z.T. nur über wenige Stunden am Tag durchgeführt (MAIER et al., 1991; HEIZMANN et al., 1987; KRÖTZL et al., 1993). Dabei besteht die Gefahr, dass die Präsenz des Beobachters das Verhalten der Tiere beeinflusst und dass ein mehr oder weniger repräsentativer Ausschnitt aus dem Verhaltensinventar erlangt wird. Verhalten findet jedoch ganzheitlich am Tage wie in der Nacht über 24 Stunden hinweg statt. Mit der Infrarot-Videotechnik und Langzeitaufnahmen ist es möglich, das Verhalten (im vorliegenden Fall die Beschäftigung mit Stroh) lückenlos zu erfassen und mittels computergestützter Verfahren (hier: OBSERVER/Video Tape Analysis System) sekundengenau auszuwerten (Literaturzusammenstellung bei HOY, 1998a, b).

Durch die Anwendung der genannten Techniken konnte nachgewiesen werden, dass Eber in Besamungsstationen sich durchschnittlich zu 106,6 Minuten in 24 Stunden mit Stroh beschäftigten. In einer Analyse von ROHRMANN und HOY (2004a) in 21 deutschen Besamungsstationen mit 4338 Buchten konnte gezeigt werden, dass die Eberbuchten zu 100 \% mit Stroheinstreu bewirtschaftet werden. Demgegenüber ist bekannt, dass z.B. in den USA, aber auch in den Niederlanden und Frankreich Eber häufig noch in Kastenständen und in einstreulosen Buchten gehalten werden (BRUININX et al., 1998; SINGLETON, 2001). In der vorliegenden Untersuchung wurde zugleich aber deutlich, dass zwischen den einzelnen Ebern ganz erhebliche Unterschiede hinsichtlich der Beschäftigung mit Stroh auftreten. Die Spannweite umfasst ein Minimum von etwa 22 Minuten und ein Maximum von nahezu 4,5 Stunden pro Tag.

Die Anwendung der univariaten Varianzanalyse erbrachte den Nachweis, dass die Besamungsstation, die Jahreszeit und die Buchtengröße einen signifikanten Einfluss auf die Beschäftigung mit Stroh haben. ROHRMANN und HOY (2004b) konnten zeigen, dass die Eber im Winter insgesamt aktiver waren und im Sommer umgekehrt die Gesamtliegedauer höher war. Die höhere Aktivität der Tiere war auch in der um 1,8 \% höheren Beschäftigungsdauer im Winter sichtbar. Vermutlich führte die z.T. deutlich höhere Stalllufttemperatur im heißen Sommer 2003 (gemessen in der Station A in einer anderen Untersuchung - BEKKERING et al., 2003) zu einer stärkeren thermischen Belastung der Tiere und somit zu einer geringeren Aktivität.

Mit zunehmender Buchtengröße stieg die Häufigkeit des Laufens in der Bucht (ROHRMANN und HOY, 2004b) und in Verbindung damit der prozentuale Zeitanteil der Beschäftigung. Allerdings muss darauf hingewiesen werden, dass der hohe Wert von 11,9 \% auf den Daten von insgesamt nur 4 Buchten mit einer nicht repräsentativen Fläche von 13,22 m² (2 x 4 Tiere - Sommer, Winter) beruht. Würden die Buchtengrößen $>7 \mathrm{~m}^{2} \leq 9 \mathrm{~m}^{2}$ sowie $>13 \mathrm{~m}^{2}$ zusammengefasst, ergäbe sich kein signifikanter Einfluss der Buchtenkategorie auf die Beschäftigung mit Stroh.

Zwischen den Besamungsstationen traten deutliche Unterschiede in der Beschäftigung mit Stroh - gemessen am Zeitanteil in 24 Stunden - auf. Bei der Suche nach den Ursachen wurden Tagesgänge für die prozentuale Beschäftigung mit der Einstreu auf Stundenbasis unter Berücksichtigung verschiedener Zeitgeber, wie Fütterung, Einstreuversorgung und Tränkzeiten (nur Station A und nur im Sommer), berechnet. Dabei wurde der höchste prozentuale Anteil an Beschäftigung im Sommer in Besamungsstation A (11,4 \% in $24 \mathrm{~h}$ ) nachgewiesen - in einer Periode, in der zweimal pro Tag gefüttert, dreimal pro Tag getränkt und einmal in drei Tagen eingestreut wurde. Aus den grafischen Darstellungen 1 bis 6 ist abzulesen, dass eine Zunahme des 
Beschäftigungsanteils pro Stunde immer in unmittelbarer zeitlicher Nähe zu einem Zeitgeber stattfand. Besonders deutlich kann dies wiederum für den Sommerdurchgang in Besamungsstation A (6 Zeitgeber) sichtbar gemacht werden (Abb. 1). Die Unterschiede in der prozentualen Häufigkeit der Beschäftigung mit Stroh zwischen den Stationen können somit im Wesentlichen auf das Management zurückgeführt werden. Dies konnte in gleicher Weise für das Liegeverhalten gezeigt werden (ROHRMANN und HOY, 2004b). Je häufiger die Besamungseber veranlasst werden, zum Fressen, zum Trinken oder bei der Einstreuversorgung aufzustehen, um so größer ist der Zeitanteil der Beschäftigung mit Stroh. Dies ist dadurch zu erklären, dass die Tiere nach dem Fressen und Trinken und vor allem nach dem Einstreuen sich intensiv mit dem Einstreumaterial beschäftigten, bevor sie sich wieder ablegten. Aus den Ergebnissen kann geschlussfolgert werden, dass die Haltung der Tiere auf Stroh per se noch keine Garantie für einen hohen Grad der Beschäftigung damit bietet. Dafür sprechen die großen individuellen Unterschiede im Liegeverhalten (manche Eber liegen bis $92 \%$ in 24 Stunden - ROHRMANN und HOY, 2004b) und im Beschäftigungsanteil pro 24 Stunden (zwischen 1,5\% und 18,6 \%) sowie die signifikanten managementbedingten Differenzen zwischen den Stationen, die sich um den Faktor 1,6 unterscheiden (Vergleich Station A zu Station C).

\section{Literatur}

BEKKERING, J.; BRANDT, H.; HOY, ST.:

Results of continuous measurements of ear temperature in boars. Proc. $1^{\text {st }}$ ECPLF (European Congress Precision Livestock Farming), Wageningen Academic Publishers (2003), 23-26

VON BORELL, E.; LENGERKEN, G. VON; RUDOVSKY, A.:

Tiergerechte Haltung von Schweinen. In: METHLING, W. und UNSELM, J. (Hrsg.): Umwelt- und tiergerechte Haltung von Nutz-, Heim- und Begleittieren Verlag Paul Parey (2002), 333-357

BRUININX, E.M.A.M.; VERMEER, H.M.; VEREIJKEN, P.F.G.; WASSENAAR, T.; SWINKELS, J.W.G.M.: Hoktype en welzijn van K.I.-beren. Praktijkonderzoek Varkenshouderij, Rosmalen (1998)

BUCHENAUER, D.:

Symptome des Wohlbefindens oder Unwohlseins beim Schwein unter Berücksichtigung intensiver Haltungsbedingungen. In: MILITZER, K.: Wege zur Beurteilung tiergerechter Haltungsbedingungen bei Labor-, Zoo- und Haustieren. Verlag Paul Parey, (1986), 54-65

GRAUVOGL, A.:

Erfüllen die Haltungssysteme die Bedürfnisse? Der Tierzüchter 39 (1987), 527-529

HEIZMANN, V.; HAUSER, C.; MANN, M.:

Zum Erkundungs- und Spielverhalten juveniler Hausschweine in der Stallhaltung. Aktuelle Arbeiten zur artgemäßen Tierhaltung. KTBL-Schrift 323 (1987), 243-265

HORSTMEYER, A.; VALLBRACHT, A.:

Artgerechte Schweinehaltung - ein Modell. Tierhaltung Band 20 (1990) Verlag Birkhäuser Basel

HOY, ST.:

Nutzung der Infrarot-Videotechnik in der angewandten Nutztierethologie. Tierärztliche Umschau 53 (1998a), 554-559

HOY, ST.:

Anwendung der computergestützten Verhaltensauswertung in der Nutztierethologie mit Hilfe des OBSERVER/Video-Tape-Analysis-Systems. Tierärztliche Umschau 53 (1998b), 10, 606-613

JACKISCH, T.; HESSE, D.; SCHLICHTING, M.:

Strohwühlen statt Schwanzbeißen. Deutsche Geflügelwirtschaft und Schweineproduktion 40 (1996), 5356

KRESS, B.; ROSS, A.; VAN DEN WEGHE, H.; STEFFENS, G.:

Stroh als Beschäftigungsmaterial in der einstreulosen Schweinemast unter besonderer Berücksichtigung des Tierverhaltens. Proc. Intern. Tagung Bau, Technik und Umwelt in der landwirtschaftlichen Nutztierhaltung 9./10.3.1999, 279-283

KRÖTZL, H.; SCIARRA, C.; TROXLER, J.:

Der Einfluß von Rauhfutterautomaten, Strohraufen und Nagebalken auf das Verhalten von Mastschweinen. Aktuelle Arbeiten zur artgemäßen Tierhaltung. KTBL-Schrift 361 (1993), 181-191 
MAIER, P.; HEIZMANN, V.; REISEN BAUER, K.:

Sozialverhalten und Verhaltensontogenese von Hausschweinen in einem möblierten Familienstall. Aktuelle Arbeiten zur artgemäßen Tierhaltung. KTBL-Schrift 351 (1991), 129-140

MARX, D.; BUCHHOLZ, M.:

Ethologische Wahlversuche mit frühabgesetzten Ferkeln während der Haltung in Buchten mit unterschiedlicher Anwendung von Stroh. 2. Mitteilung: Auswirkungen verschiedener Anwendungen des Strohs bei unterschiedlichen Flächengrößen. Dtsch. tierärztl. Wschr. 93 (1991), 75-80

ROHRMANN, ST.; HOY, ST.:

Untersuchungen zum Verhalten von Ebern in Besamungsstationen. Aktuelle Arbeiten zur artgemäßen Tierhaltung. KTBL-Schrift (2004a) im Druck

ROHRMANN, ST.; HOY, ST.:

Analyse der Körpermaße und Haltungsbedingungen von Besamungsebern unter Tierschutzaspekten. Proc. 9. Intern. Fachtagung Tierschutz 19./20.2.2004b, im Druck

SINGLETON, W.L.:

State of the art in artificial insemination of pigs in the United States. Theriogenology 56 (2001), 13051310

STUBBE, A.:

Entwicklung und Beurteilung einer Beschäftigungsmöglichkeit für Mastschweine in intensiven Haltungssystemen. Diss. Univ. Hohenheim 2000

WECHSLER, B.:

Schwein. In: Das Buch vom Tierschutz (Hrsg.: H.H. SAMBRAUS und A. STEIGER) Enke-Verlag 1997

Eingegangen: 15.03.2004

Akzeptiert: 23.09.2004

Autor für Korrespondenz

Prof. Dr. STEFFEN HOY

Justus-Liebig-Universität Gießen

Institut für Tierzucht und Haustiergenetik

Bismarckstraße 16

D-35390 Gießen 